\title{
Magnetoresistance in multilayer fullerene spin valves: A first-principles study
}

\author{
Deniz Çakır, ${ }^{1, *}$ Diana M. Otálvaro, ${ }^{2, \dagger}$ and Geert Brocks ${ }^{2, \ddagger}$ \\ ${ }^{1}$ Department of Physics, University of Antwerp, Groenenborgerlaan 171, B-2020 Antwerpen, Belgium \\ ${ }^{2}$ Computational Materials Science, Faculty of Science and Technology and MESA+ Institute for Nanotechnology, University of Twente, \\ P.O. Box 217, 7500 AE Enschede, The Netherlands
}

(Received 23 October 2014; revised manuscript received 20 November 2014; published 2 December 2014)

\begin{abstract}
Carbon-based molecular semiconductors are explored for application in spintronics because their small spinorbit coupling promises long spin lifetimes. We calculate the electronic transport from first principles through spin valves comprising bi- and tri-layers of the fullerene molecules $\mathrm{C}_{60}$ and $\mathrm{C}_{70}$, sandwiched between two $\mathrm{Fe}$ electrodes. The spin polarization of the current, and the magnetoresistance depend sensitively on the interactions at the interfaces between the molecules and the metal surfaces. They are much less affected by the thickness of the molecular layers. A high current polarization $(\mathrm{CP}>90 \%)$ and magnetoresistance $(\mathrm{MR}>100 \%)$ at small bias can be attained using $\mathrm{C}_{70}$ layers. In contrast, the current polarization and the magnetoresistance at small bias are vanishingly small for $\mathrm{C}_{60}$ layers. Exploiting a generalized Jullière model we can trace the differences in spin-dependent transport between $\mathrm{C}_{60}$ and $\mathrm{C}_{70}$ layers to differences between the molecule-metal interface states. These states also allow one to interpret the current polarization and the magnetoresistance as a function of the applied bias voltage.
\end{abstract}

DOI: 10.1103/PhysRevB.90.245404

PACS number(s): 72.25.Mk, 73.40.Sx, 75.47.De, 75.78.-n

\section{INTRODUCTION}

Spintronics focuses on information processing with charge carrier spins [1]. Developments in spintronics, such as giant magnetoresistance (GMR) and tunneling magnetoresistance (TMR) in metallic spin valves have revolutionized the fields of magnetic recording and storage. Novel devices are envisioned that use injection and manipulation of spin-polarized currents in semiconductors, such as the spin transistor [2]. Molecular semiconductors (MSC), i.e., semiconductors comprising organic molecules, have drawn much attention because carbon-based molecules promise to have advantages over conventional semiconductors such as $\mathrm{Si}$ or GaAs $[3,4]$. The relatively weak spin-orbit coupling and hyperfine interactions in such molecules lead to long spin life times, i.e., long spin relaxation and dephasing times, which would allow for robust spin operations and read-out, provided the limited charge carrier mobility that is typical for MSCs does not present a bottleneck. The use of molecules also opens up a route towards single molecule spintronics, where individual molecules are considered for electronic functions. Indeed, magnetoresistance effects have been demonstrated at the single molecule level [5-8].

Many experimental studies deal with vertical spin valves, where molecular layers are sandwiched between two ferromagnetic metal (FM) electrodes, and are used either as a tunnel barrier, or as charge and spin transport medium. Large magnetoresistance (MR) effects have been reported in spin valves based upon layers of organic molecules such as tris(8hydroxy-quinolinato)-aluminium $\left(\mathrm{Alq}_{3}\right)$ [9-15], or fullerenes such as $\mathrm{C}_{60}$ [16-20]. Similar effects have been observed in zinc methyl phenalenyl (ZMP) layers sandwiched between an FM electrode and a nonmagnetic electrode, where the spin

\footnotetext{
*deniz.cakir@uantwerpen.be

†d.otalvaro@utwente.nl

${ }^{\ddagger}$ g.h.l.a.brocks@utwente.nl
}

valve effect has been attributed to the special characteristics of the molecule/FM interface layer [21]. In phenomenological models for the observed spin transport effects the electronic structure, in particular the spin polarization, of the MSC/FM interfaces plays a pivotal role in spin injection into the MSC [14]. This has prompted the suggestion that highly spin-polarized currents in spintronic devices may be obtained by exploiting such interface interactions, which has been dubbed "spinterface science" [22], and has motivated research into the role played by the interfaces [23-29].

The electronic structure of metal-organic interfaces is accessible through first-principles calculations, and can in some cases be interpreted using simple models for the energy level line-up at interfaces [30-32]. Photoemission spectroscopy or scanning tunneling microscopy, combined with first-principles calculations, enable a detailed analysis of the spin-dependent electronic properties of metal-organic interfaces. Bonding between a molecule and a ferromagnetic metal leads to spin-split (anti)bonding states and induces a spin polarization that extends onto the molecule [6,33-39]. For instance, calculations on $\mathrm{C}_{60} \mid \mathrm{Fe}(001)$ interfaces yield a magnetic moment of $0.2 \mu_{B}$ induced on the $\mathrm{C}_{60}$ molecules [38]. For electronic transport in spintronics devices, not the overall spin polarization is decisive, however, but the spin polarization of the states around the Fermi energy.

First-principles transport calculations might establish the connection between such molecule-metal interface states and MR effects in molecular spin valves. Calculations have been applied to model currents through a single molecule attached to two FM metal electrodes [40-44], as they can be realized in STM experiments, for instance, where MR effects have been demonstrated at the single molecule level [5-8]. A single molecule is, however, not a good starting point for modeling transport through MSCs, as binding a molecule to two electrodes markedly changes its electronic structure. For instance, fullerene molecules attached to two Fe electrodes result in metallic conduction, whereas fullerene multilayers give a small, tunneling conductance [17]. 
We have calculated the spin-dependent transport through multilayer graphene spin valves $[45,46]$, and recently have demonstrated the feasibility of such calculations on molecular spin valves [47]. In this paper we expand the scope of such calculations. In particular we focus on $\mathrm{Fe} \mid$ fullerene $\mid \mathrm{Fe}$ spin valves with bi- and tri-layers of $\mathrm{C}_{60}$ and $\mathrm{C}_{70}$ fullerene molecules. Fullerenes are particularly interesting molecules for applications in spintronics due to the absence of hydrogen atoms that lead to spin dephasing via hyperfine interactions. The $\mathrm{Fe}(001)$ surface is a well-established substrate for organic spintronics allowing for a controlled growth of fullerene layers $[36,38]$. We study the links between the spin-dependent transport through these fullerene multilayers, and the electronic structure of the metal-organic interfaces by first-principles transport calculations. A generalized Jullière or factorization model, defined in Sec. II, serves to rationalize these links, in particular when a single molecular state dominates the transport.

The setup of the transport calculations is discussed in Sec. III, and the results are discussed in Sec. IV. Somewhat surprisingly, there is a qualitative difference between the spin transport through $\mathrm{C}_{60}$ and $\mathrm{C}_{70}$ layers, which can be traced to a difference in the molecule-metal interface states. In particular, adsorption of $\mathrm{C}_{70}$ leads to a spin-polarized interface state very close to the Fermi level that gives rise to a large current polarization $(\mathrm{CP})$ and $\mathrm{MR}$. In contrast, the corresponding interface state associated with adsorption of $\mathrm{C}_{60}$ lies further from the Fermi level. That state is accessible by increasing the bias voltage over the spin valve, which however, only leads to a relatively moderate $\mathrm{CP}$ and MR. Section V summarizes the main conclusions.

\section{THEORY}

Following Landauer, the current through a quantum conductor $I^{\sigma}$ at finite bias $V$ and zero temperature, carried by independent particles with spin $\sigma=\uparrow, \downarrow$, is given by [48]

$$
I^{\sigma}=\frac{e}{h} \sum_{\sigma} \int_{E_{F}-\frac{1}{2} e V}^{E_{F}+\frac{1}{2} e V} T^{\sigma}(E, V) d E,
$$

with $T^{\sigma}$ the transmission probability of an electron with spin $\sigma$. Expressed in nonequilibrium Green's functions (NEGF) [49,50],

$$
T^{\sigma}=\operatorname{Tr}\left[\boldsymbol{\Gamma}_{R}^{\sigma} \mathbf{G}_{R L}^{\sigma, r} \boldsymbol{\Gamma}_{L}^{\sigma} \mathbf{G}_{L R}^{\sigma, a}\right],
$$

where $\mathbf{G}_{R L}^{\sigma, r}$ is the block of the retarded Green's function matrix connecting the right and left leads through the quantum conductor, $\boldsymbol{G}_{L R}^{\sigma, a}=\left(\mathbf{G}_{R L}^{\sigma, r}\right)^{\dagger}$ is the corresponding advanced Green's function matrix block, and $\Gamma_{R(L)}^{\sigma}=-2 \operatorname{Im} \Sigma_{R(L)}^{\sigma}$, with $\Sigma_{R(L)}^{\sigma}$ the self-energy matrix connecting the quantum conductor to the ideal right (left) lead [48,50,51].

One can rewrite this expression by formally partitioning the system into a right and a left part and a coupling between the parts. A natural partitioning for organic spin valves is a left and a right interface, each consisting of a molecular layer adsorbed on a metal surface [47]. Any molecular layers between the two interfaces are then incorporated in the coupling Hamiltonian.

In the tunneling regime, where the effects of multiple reflections between left and right parts can be neglected, it is possible to simplify the transmission to

$$
T^{\sigma}=4 \pi^{2} \sum_{i, j} n_{R i}^{\sigma} n_{L j}^{\sigma}\left|H_{R i, L j}^{\sigma}\right|^{2} ;
$$

see Appendix A. Here $n_{R i}^{\sigma}$ and $n_{L j}^{\sigma}$ are the spectral densities corresponding to states $i$ and $j$ of the right and left part, respectively [48,52]. The matrices $\mathbf{H}_{R L}^{\sigma}=\left(\mathbf{H}_{L R}^{\sigma}\right)^{\dagger}$ represent the coupling between the right and left parts.

Equation (3) can be used to derive a generalized Jullière expression for the magnetoresistance of an organic spin valve, if additional approximations regarding the spectral densities and the coupling matrix elements are made. If the magnetization of the right electrode is reversed when switching from parallel (P) to antiparallel (AP) configuration, it is reasonable to expect that the spectral densities of majority and minority spins are only interchanged, but not altered,

$$
\left(n_{R i}^{\sigma}\right)_{\mathrm{AP}} \approx\left(n_{R i}^{-\sigma}\right)_{P} .
$$

Following a simple tight-binding argument, the coupling matrix elements in the tunneling regime scale with the overlap between the wave functions of the left and right parts, which roughly scales as the product of these functions. If this is the case, then a decent approximation for the coupling matrix elements in the AP case should be

$$
\left|H_{R i, L j}^{\sigma}\right|_{\mathrm{AP}}^{2} \approx\left|H_{R i, L j}^{\sigma} H_{R i, L j}^{-\sigma}\right|_{P} .
$$

Using the approximations of Eqs. (4) and (5) one can express the normalized difference $\Delta_{P / \mathrm{AP}}=\left(T_{P}-T_{\mathrm{AP}}\right) /\left(T_{P}+T_{\mathrm{AP}}\right)$ between the transmissions $T=\sum_{\sigma} T^{\sigma}$ in the $\mathrm{P}$ and AP cases as

$$
\Delta_{P / \mathrm{AP}}=\frac{\sum_{i, j}\left(v_{R i, L j}^{\uparrow}-v_{R i, L j}^{\downarrow}\right)\left(v_{L j, R i}^{\uparrow}-v_{L j, R i}^{\downarrow}\right)}{\sum_{i, j}\left(v_{R i, L j}^{\uparrow}+v_{R i, L j}^{\downarrow}\right)\left(v_{L j, R i}^{\uparrow}+v_{L j, R i}^{\downarrow}\right)},
$$

in terms of the weighted densities,

$$
v_{R i, L j}^{\sigma}=n_{R i}^{\sigma}\left|H_{R i, L j}^{\sigma}\right| ; v_{L j, R i}^{\sigma}=n_{L j}^{\sigma}\left|H_{R i, L j}^{\sigma}\right|,
$$

which are calculated for the $\mathrm{P}$ case. Equation (6) has the form of a generalized Jullière expression in terms of weighted spinpolarization densities [53].

The expression can be simplified even further if the spectral density of each spin is dominated by a single state. For adsorbed molecules this is likely to be the case for an energy range close to one particular molecular level, the HOMO or LUMO, for instance. The sum $\sum_{i, j}$ in Eq. (6) is then over one state, giving $\Delta_{P / \mathrm{AP}}=P_{R} P_{L}$ with $P_{R}=$ $\left(v_{R, L}^{\uparrow}-v_{R, L}^{\downarrow}\right) /\left(v_{R, L}^{\uparrow}+v_{R, L}^{\downarrow}\right)$ the weighted spin-polarization density of the right interface, where $v_{R, L}^{\sigma}=n_{R}^{\sigma}\left|H_{R L}^{\sigma}\right|$, and $P_{L}$ a similar expression for the spin-polarization density of the left interface. In linear response, where the bias $V$ in Eq. (1) is infinitesimal, only the transmissions at the Fermi energy are important. The magnetoresistance then becomes $\mathrm{MR}=\left(T_{\mathrm{AP}}-T_{P}\right) / T_{\mathrm{AP}}=2 P_{L} P_{R} /\left(1-P_{L} P_{R}\right)$, which has the appearance of a Jullière expression [53].

Assuming that a single state is dominant also allows for simplifying the transmission of Eq. (3) to $T^{\sigma}=4 \pi^{2} n_{R}^{\sigma} n_{L}^{\sigma}\left|H_{R L}^{\sigma}\right|^{2}$. For a symmetric junction in the $\mathrm{P}$ configuration at zero bias, one has $n_{L}^{\sigma}=n_{R}^{\sigma}$, and thus $\sqrt{T_{P}^{\sigma}}=n_{R}^{\sigma}\left|H_{R L}^{\sigma}\right|$, linking the transmission directly to the interface density of states $n_{R}$. Applying a bias voltage $V$ across a tunnel barrier, it is 
(a)

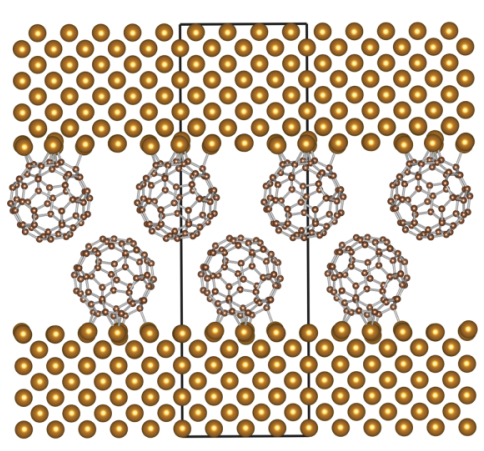

(b)

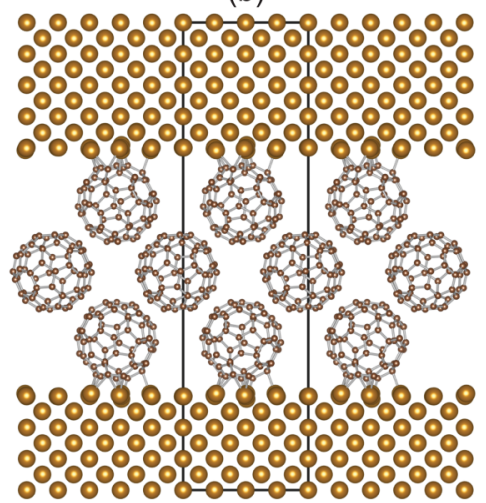

(c)

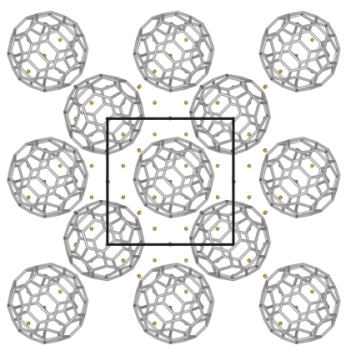

FIG. 1. (Color online) (a) and (b) Side views of the bilayer and trilayer $\mathrm{C}_{60}$ junctions, $\mathrm{Fe}\left|\left(\mathrm{C}_{60}\right)_{n}\right| \mathrm{Fe}, n=2,3$; (c) top view of interlayer $\mathrm{C}_{60}$ stacking. The black lines denote the supercell used.

reasonable to assume that the small transmission current does not change the charge distribution. The spectral densities of the right and left interfaces can then be obtained from rigid shifts of the corresponding densities at zero bias, $n_{R i}^{\sigma}(E, V)=$ $n_{R i}^{\sigma}(E-\mathrm{eV} / 2,0)$ and $n_{L j}^{\sigma}(E, V)=n_{L j}^{\sigma}(E+\mathrm{eV} / 2,0)$. Again assuming that at each energy a single state is dominant (not necessarily the same state at all energies), it then follows:

$$
T_{P}^{\sigma}(E, V) \approx \sqrt{T_{P}^{\sigma}\left(E-\frac{e V}{2}, 0\right)} \sqrt{T_{P}^{\sigma}\left(E+\frac{e V}{2}, 0\right)},
$$

and

$$
T_{\mathrm{AP}}^{\sigma}(E, V) \approx \sqrt{T_{P}^{\sigma}\left(E-\frac{e V}{2}, 0\right)} \sqrt{T_{P}^{-\sigma}\left(E+\frac{e V}{2}, 0\right)} .
$$

With these expressions one can interpret the transmission spectra at any bias, starting from the spectrum of the $\mathrm{P}$ case at zero bias. These factorization approximations are valid if a single state dominates (not necessarily the same state at each energy). They lead to a positive MR for symmetric junctions; negative MR for such junctions can occur if multiple states interact, as we will discuss in Sec. IV A.

\section{COMPUTATIONAL DETAILS}

The $\mathrm{Fe}(001) \mid$ fullerene interfaces are modeled by a $4 \times 4$ $\mathrm{Fe}(001)$ surface unit cell, with a cell parameter of $11.32 \AA$, containing one fullerene molecule (see Fig. 1). For comparison, the nearest neighbor distance in $\mathrm{C}_{60}$ and $\mathrm{C}_{70}$ crystals is $10-11 \AA[54,55]$. From a number of possible adsorption geometries, we have identified the most stable structures of adsorbed $\mathrm{C}_{60}$ and $\mathrm{C}_{70}$ molecules. Details can be found in Appendix B.

We optimize the structures of the $\mathrm{Fe}(001) \mid$ fullerene interfaces within density functional theory (DFT), using projector augmented waves (PAW) [56,57], as implemented in the Vienna Ab Initio Simulation Package (VASP) [58,59]. All plane waves up to a kinetic energy cutoff of $400 \mathrm{eV}$ are included in the basis set. The spin-polarized PBE functional is used to describe exchange and correlation [60]. As the bonding between the Fe surface and the fullerene molecules is strong, including van der Waals interactions is not necessary. An equidistant $k$-point grid with a spacing of $0.02 \AA^{-1}$ is used for the Brillouin zone sampling. Structures are assumed to be relaxed when the difference of the total energies between two consecutive ionic steps is less than $10^{-5} \mathrm{eV}$ and the maximum force on each atom is less than $0.01 \mathrm{eV} / \AA$.

A structure for a bilayer- $\mathrm{C}_{60}$ junction, $\mathrm{Fe}\left|\left(\mathrm{C}_{60}\right)_{2}\right| \mathrm{Fe}$, is generated by mirroring the optimized $\mathrm{Fe}(001) \mid \mathrm{C}_{60}$ interface structure, and translating it in plane by half a lattice constant, such that the packing $\mathrm{C}_{60}$ molecules in the bilayer resembles that of the (001) orientation of the fcc $\mathrm{C}_{60}$ crystal (see Fig. 1). The spacing between the $\mathrm{C}_{60}$ layers is chosen such that the shortest intermolecular $\mathrm{C}-\mathrm{C}$ distance is $3.2 \AA$, which is a typical value for close-packed fullerenes or carbon nanotubes. Along the same lines a structure for a trilayer $\mathrm{C}_{60}$ junction, $\mathrm{Fe}\left|\left(\mathrm{C}_{60}\right)_{3}\right| \mathrm{Fe}$, is generated, as well as structures for bi- and trilayer $\mathrm{C}_{70}$ junctions, $\mathrm{Fe}\left|\left(\mathrm{C}_{70}\right)_{n}\right| \mathrm{Fe}, n=2,3$.

Electronic transport in $\mathrm{Fe} \mid$ fullerene|Fe junctions is calculated using the self-consistent NEGF technique, Eqs. (1) and (2), as implemented in TRANSIESTA [50,61]. We employ Troullier-Martins (TM) norm-conserving pseudopotentials (NCPP) [62], the PBE functional, and an energy cutoff for the real-space mesh of 200 Ry. Numerical orbital basis sets are used, comprising single $\zeta$ and double $\zeta$ plus polarization for Fe and C, respectively. To compare the VASP and SIESTA results, we benchmark the calculations on the magnetic properties of bulk bcc Fe and the clean Fe(001) surface (see Appendix B).

Using a $6 \times 6$ in-plane $k$-point mesh in the $4 \times 4 \mathrm{Fe}(001)$ supercell suffices to obtain converged results for the transmission, as is demonstrated by Fig. 2 .

\section{RESULTS AND DISCUSSION}

\section{A. Linear response}

The conductance in the linear response regime is determined by the transmission at the Fermi level. Table I gives the calculated transmissions of bilayers and trilayers of $\mathrm{C}_{60}$ and $\mathrm{C}_{70}$ molecules, sandwiched between two $\mathrm{Fe}(001)$ electrodes, with magnetizations parallel (P) or antiparallel (AP). The transmission through a trilayer is up to two orders of magnitude smaller than the transmission through a bilayer. In absolute numbers the transmission through a bilayer is fairly high; The small numbers obtained for a trilayer are typical for the 


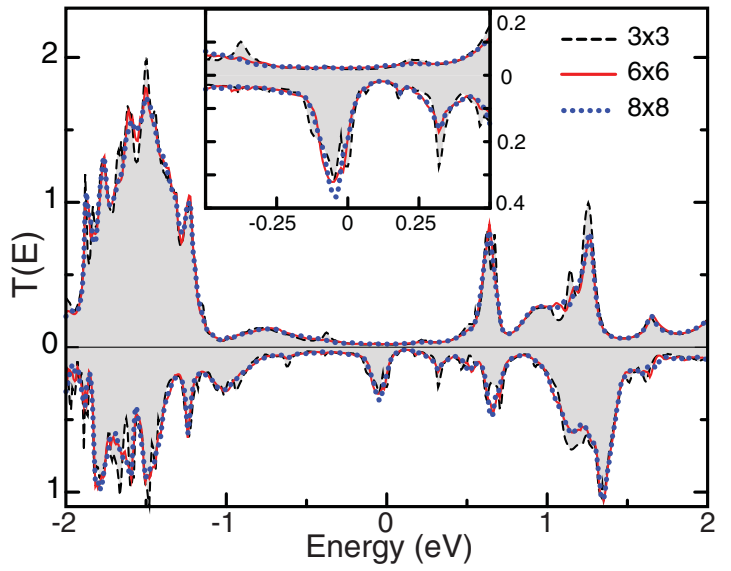

FIG. 2. (Color online) Transmissions $T_{P}^{\uparrow}(E)$ of majority (top) and $T_{P}^{\downarrow}(E)$ (bottom) of minority spin channels of $\mathrm{Fe}\left|\left(\mathrm{C}_{70}\right)_{2}\right| \mathrm{Fe}$ at zero bias. The Fermi level is at zero energy. Curves are given for $3 \times 3,6 \times 6$, and $8 \times 8 k$-point grid samplings.

tunneling regime. The sizable difference in the transmissions of bi- and trilayers shows that we are not in the regime of resonant transmission through a molecular level.

The transmissions of $\mathrm{C}_{70}$ bi- or trilayers are consistently higher than that of their $\mathrm{C}_{60}$ counterparts, although the relevant energy levels and wave functions (HOMO and LUMO) of the isolated $\mathrm{C}_{60}$ and $\mathrm{C}_{70}$ molecules are not so different. Below we will argue that the difference in transmission is caused by differences in the states formed at the $\mathrm{Fe} /$ molecule interfaces.

The most prominent difference between $\mathrm{C}_{60}$ and $\mathrm{C}_{70}$ molecules is in the spin polarization of the transmission. Whereas for $\mathrm{C}_{60}$ layers the transmissions of majority and minority spins are almost equal; for $\mathrm{C}_{70}$ layers the transmission of minority spin is approximately an order of magnitude larger than that of majority spin. It means that the current polarization in linear response, $\mathrm{CP}=\left(T_{P}^{\uparrow}-T_{P}^{\downarrow}\right) /\left(T_{P}^{\uparrow}+T_{P}^{\downarrow}\right)$, of $\mathrm{C}_{60}$ junctions at low bias is small, $|\mathrm{CP}|<20 \%$. In contrast, the $\mathrm{CP}$ of $\mathrm{C}_{70}$ junctions is very substantial, $|\mathrm{CP}|=80 \%-90 \%$. Moreover the magnetoresistance $\mathrm{MR}=\left(T_{P}-T_{\mathrm{AP}}\right) / T_{\mathrm{AP}}$ is large for $\mathrm{C}_{70}$ junctions, exceeding $100 \%$ for trilayers, whereas the $\mathrm{MR}$ for $\mathrm{C}_{60}$ junctions is vanishingly small. The differences between the $\mathrm{CP}$ and $\mathrm{MR}$ of $\mathrm{C}_{60}$ and $\mathrm{C}_{70}$ junctions have the same origin, as we will see below.

In the following we interpret the behavior of $\mathrm{C}_{60}$ and $\mathrm{C}_{70}$ junctions using the model outlined in Sec. II. If a single channel dominates the transmission and the junction is symmetric, then transmission can be factorized according to Eqs. (8) and (9),
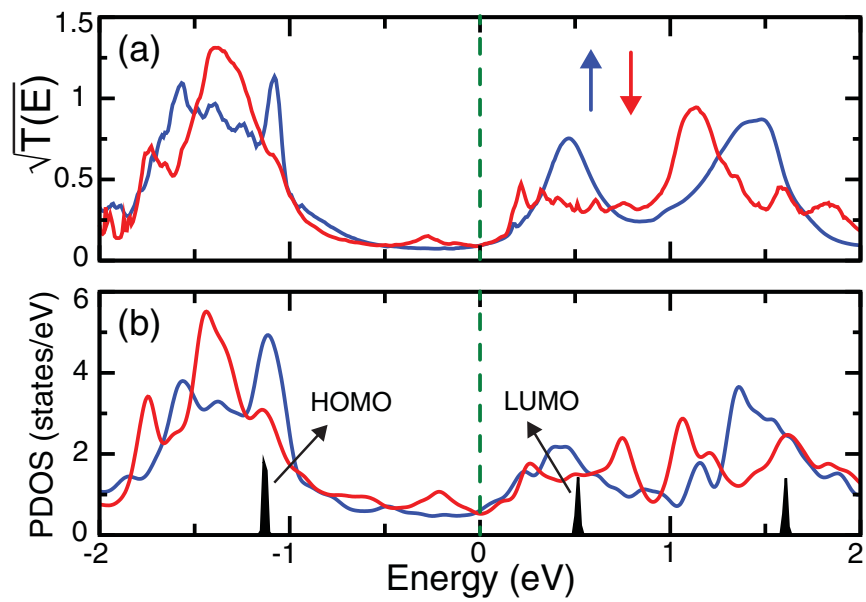

FIG. 3. (Color online) (a) $\sqrt{T_{P}^{\uparrow}(E)}$ for majority spin (blue) and $\sqrt{T_{P}^{\downarrow}(E)}$ for minority spin (red) at zero bias for $\mathrm{Fe}\left|\mathrm{C}_{60}-\mathrm{C}_{60}\right| \mathrm{Fe}$ junction; (b) projected density of states (PDOS) of the $\mathrm{Fe} \mid \mathrm{C}_{60}$ interface.

and the factors $\sqrt{T_{P}^{\sigma}}=n_{R}^{\sigma}\left|H_{R L}^{\sigma}\right|$, are weighted interface density of states. Figure 3(a) shows $\sqrt{T_{P}^{\sigma}(E)}$, derived from the transmission spectra of a bilayer $\mathrm{C}_{60}$ junction. For comparison, Fig. 3(b) shows the density of states of a $\mathrm{C}_{60} / \mathrm{Fe}(001)$ interface, projected on the molecule (PDOS); see Appendix B. There is indeed a striking resemblance between the transmission spectra and the PDOSs.

The peaks in the PDOS can be labeled according to their molecular character. As the molecule-substrate interaction is large, these peaks correspond to hybrid interface states, which are significantly broadened in energy compared to the pure molecular states. Moreover, the interface states are exchange split because the substrate is ferromagnetic. Nevertheless the dominant components of their molecular contributions can still be identified; details can be found in Appendix B.

The Fermi level is situated in a gap in the transmission spectra of the bilayer $\mathrm{C}_{60}$ junction, which according to the PDOS and the molecular level spectrum corresponds to the HOMO-LUMO gap. One has $T_{P}^{\uparrow}\left(E_{F}\right) \approx T_{P}^{\downarrow}\left(E_{F}\right)$, and this absence of spin polarization is also observed in the PDOS. It is then not surprising to find that $\mathrm{CP} \approx 0$ and $\mathrm{MR} \approx 0$ at low bias. The two small peaks in the minority spin transmission $T_{P}^{\downarrow}(E)$ at $E \approx E_{F} \pm 0.2 \mathrm{eV}$ will give rise to a moderate nonzero $\mathrm{CP}$ and MR at finite bias, as we will see in the next section. These peaks are derived from hybridizing the $\mathrm{Fe}(001)$ surface states with the $\mathrm{C}_{60}$ LUMO.

TABLE I. Transmissions $T_{P}^{\uparrow(\downarrow)}$ of majority (minority) spins through Fe|layer|Fe at zero bias, magnetizations of electrodes parallel; transmission $T_{\mathrm{AP}}^{\uparrow(\downarrow)}$, magnetizations antiparallel; current polarization $\mathrm{CP}=\left(T_{P}^{\uparrow}-T_{P}^{\downarrow}\right) /\left(T_{P}^{\uparrow}+T_{P}^{\downarrow}\right)$, normalized magnetoresistance $\Delta_{P} / \mathrm{AP}=$ $\left(T_{P}-T_{\mathrm{AP}}\right) /\left(T_{P}+T_{\mathrm{AP}}\right)$, and optimistic magnetoresistance $\mathrm{MR}=\left(T_{P}-T_{\mathrm{AP}}\right) / T_{\mathrm{AP}}($ in $\%)$.

\begin{tabular}{lcccccc}
\hline \hline Layer & $T_{P}^{\uparrow}\left(E_{F}\right)$ & $T_{P}^{\downarrow}$ & $T_{\mathrm{AP}}^{\uparrow \downarrow}$ & $\sqrt{T_{P}^{\uparrow} T_{P}^{\downarrow}}$ & $\mathrm{CP}$ & $\Delta_{P / \mathrm{AP}}$ \\
\hline$\left(\mathrm{C}_{60}\right)_{2}$ & $8.6(-3)^{\mathrm{a}}$ & $8.4(-3)$ & $9.1(-3)$ & $8.5(-3)$ & 1.3 & -3.6 \\
$\left(\mathrm{C}_{60}\right)_{3}$ & $2.1(-4)$ & $2.9(-4)$ & $2.5(-4)$ & $2.5(-4)$ & -16 & 1.1 \\
$\left(\mathrm{C}_{70}\right)_{2}$ & $2.3(-2)$ & $1.9(-1)$ & $6.3(-2)$ & $6.6(-2)$ & -78 & 2.9 \\
$\left(\mathrm{C}_{70}\right)_{3}$ & $2.9(-4)$ & $4.1(-3)$ & $9.6(-4)$ & $11.0(-4)$ & -87 & 42 \\
\hline \hline
\end{tabular}

a $8.6 \times 10^{-3}$ 


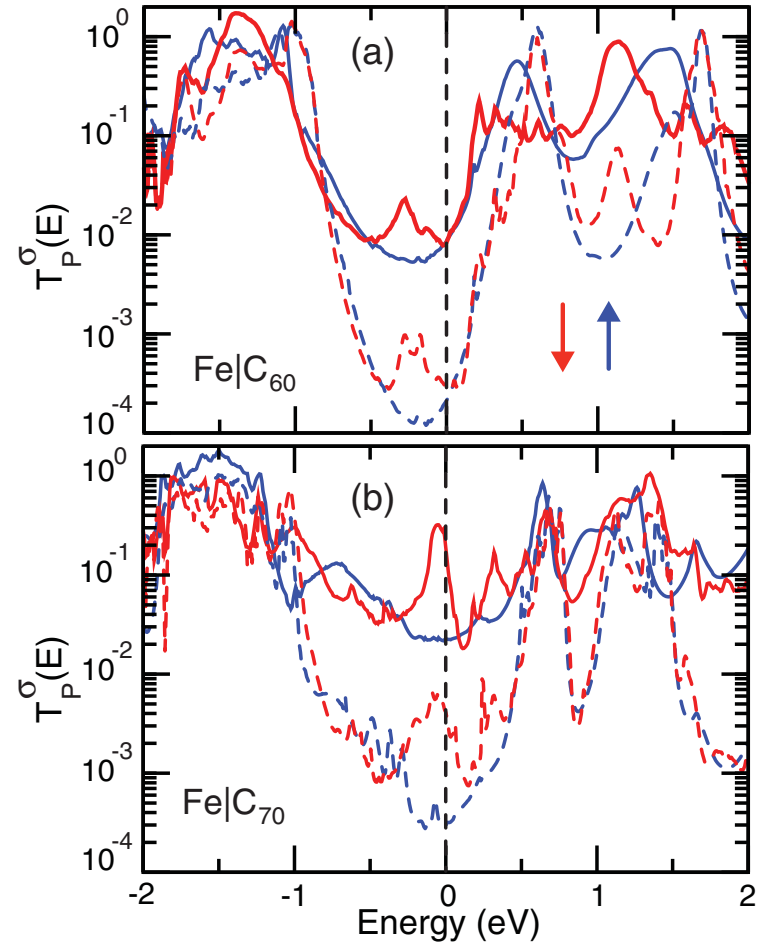

FIG. 4. (Color online) (a) Calculated transmission spectra $T_{P}^{\sigma}(E)$ of majority (blue) and minority (red) spins of bilayer $\mathrm{C}_{60}$ (solid lines) and trilayer $\mathrm{C}_{60}$ (dashed lines) junctions. The Fermi level $E_{F}$ is at zero energy. (b) Calculated transmission spectra of $\mathrm{C}_{70}$ junctions.

Figure 4 gives the transmission spectra $T_{P}^{\sigma}(E)$ of all the multilayers studied in this paper. The peaks in the transmission spectra of order unity correlate with resonant transmission through molecular levels. In the bilayer case the latter are strongly hybridized with the Fe surface, resulting in broad peaks. The transmission spectra for bi- and trilayers are qualitatively similar, but for the trilayer the peaks in the transmission are considerably sharper. For the trilayer transmission of order unity can only be achieved via resonant transmission through the molecular levels of the middle layer.

The transmission for energies in the gaps between the peaks imply tunneling through the molecular layers. In all cases the Fermi level is situated in the gap in the transmission spectrum corresponding to the molecular HOMO-LUMO gap. The transmission for energies inside this gap is higher for $\mathrm{C}_{70}$ layers than for $\mathrm{C}_{60}$ layers. This is consistent with the difference between these molecules regarding the spatial extent of their interface states. The interaction between $\mathrm{C}_{70}$ and the $\mathrm{Fe}(001)$ surface gives interface states that are more delocalized over the molecules (see Appendix B). Such a delocalization effectively leads to thinner tunnel barriers.

The most prominent difference between $\mathrm{C}_{60}$ and $\mathrm{C}_{70}$ in the transmission close to the Fermi level is a peak in the minority spin channel [compare Figs. $4(\mathrm{a})$ and $4(\mathrm{~b})$ ]. For $\mathrm{C}_{70}$ a prominent peak in the transmission is situated very close to the Fermi level, whereas for $\mathrm{C}_{60}$ a smaller peak lies at $\sim 0.2 \mathrm{eV}$ below the Fermi level. Both these peaks can be traced to an interface state derived from the molecular LUMO, created by the adsorption of the molecules on the surface. Differences in the bonding of the two molecules to the $\mathrm{Fe}(001)$ surface

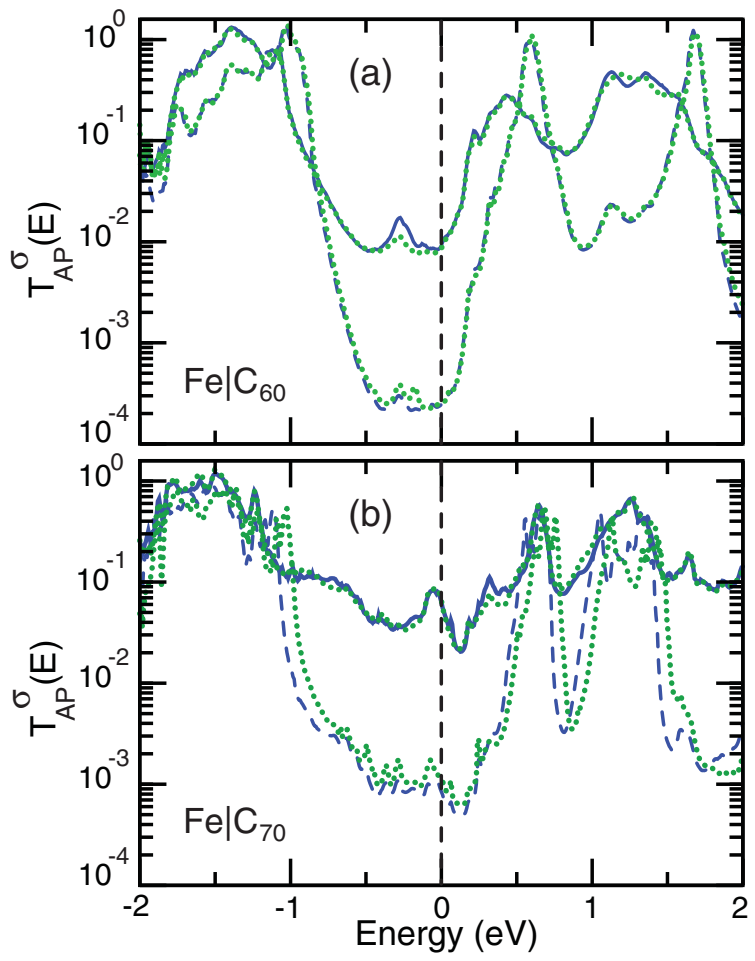

FIG. 5. (Color online) Calculated transmission spectra $T_{\mathrm{AP}}^{\sigma}(E)$ (blue) of bilayer (solid lines) and trilayer (dashed lines) molecular junctions of (a) $\mathrm{C}_{60}$ and (b) $\mathrm{C}_{70}$ [63]. The Fermi level $E_{F}$ is at zero energy. The green dotted lines give the factorization approximation $2 \sqrt{T_{P}^{\sigma}(E) T_{P}^{-\sigma}(E)}$ [see Eq. (9)].

give a different energy for this state, which has a major effect on the spin transport properties of the molecular layers (see Table I). For $\mathrm{C}_{70}$ this minority spin state at the Fermi level is at the origin of a large $\mathrm{CP}$ and a large $\mathrm{MR}$, whereas for $\mathrm{C}_{60}$ the fact that this state is not exactly at the Fermi level results in a small CP and a small MR. Going from two to three layers the transmission in the HOMO-LUMO gap decreases, but the overall pattern of the transmission remains the same.

Figure 5 shows the transmission spectra $T_{\mathrm{AP}}^{\sigma}(E)$ calculated with the magnetizations of the two Fe electrodes in antiparallel configurations. Also shown are the results of the factorization approximation, Eq. (9), with $V=0$. Following the discussion in Sec. II this approximation is designed for the tunneling regime, when multiple reflections are absent, and when a single channel dominates the transmission. The results shown in Fig. 5 seem to indicate that the factorization approximation has a somewhat wider applicability, and also works reasonably well if the transmission is larger than is typical for tunneling. From the factorization approximation it becomes clear that the $\mathrm{CP}$ and the $\mathrm{MR}$ are related properties. If the $\mathrm{CP}$ is large (small), then the MR is large (small).

There are, of course, situations where the factorization approximation fails. For instance, it always gives an MR $\geqslant 0$ for a symmetric junction in the linear response regime. This is easy to see from the discussion following Eqs. (6) and (7). In a symmetric junction at zero bias, the weighted spin polarizations of left and right interfaces are identical, $P_{R}=$ $P_{L}$, which implies that $\Delta_{P / \mathrm{AP}} \geqslant 0$ and $\mathrm{MR} \geqslant 0$. The small 
negative $\mathrm{MR}$ at zero bias calculated for a bilayer $\mathrm{C}_{60}$ junction in Table I is clearly in disagreement with this. By construction this junction is symmetric, and the right $\mathrm{Fe}(001) \mid \mathrm{C}_{60}$ interface is identical to the left interface.

Nevertheless it is possible to obtain a negative MR, even for a symmetric junction. To obtain $\Delta_{P / A P}<0$ in Eq. (6), giving $\mathrm{MR}<0$, (at least) two molecular states at each interface should be involved. In the absence of any off-diagonal coupling, i.e., $H_{R i, L j}^{\sigma}=0 ; i \neq j$, this would still give $\Delta_{P / \mathrm{AP}} \geqslant 0$, so in order to obtain a negative sign one needs a significant off-diagonal coupling. Suppose for simplicity that $H_{R i, L j}^{\sigma}=h$, $i \neq j$, and $H_{R i, L i}^{\sigma}=0$, then in a system with two states it suffices to have $n_{1}^{\downarrow}>n_{1}^{\uparrow}$ and $n_{2}^{\downarrow}<n_{2}^{\uparrow}$ to obtain $\Delta_{P / \text { AP }}<0$. In other words, a negative MR in a symmetric junction can be obtained if a strong coupling between two states exists, where one of the states has a dominant majority spin character at the Fermi level, and the other one has a dominant minority spin character.

As in the bilayer $\mathrm{C}_{60}$ junction the Fermi level falls between peaks in the transmission spectra and in the PDOS (see Fig. 3) it is quite likely that the tails of more than one molecular state are involved at this energy. The fact that in the trilayer $\mathrm{C}_{60}$ junction the negative MR disappears, shows that the coupling between these states across the junction is crucial. Despite its limitations, the factorization model can be very helpful in interpreting spin transport properties as we will see in the next section.

\section{B. Finite bias}

Figure 6(a) shows the magnetoresistance (MR) as a function of the applied bias $V$, calculated self-consistently for the Fe|bilayer $\mathrm{C}_{60} \mid \mathrm{Fe}$ junction. Over the voltage range studied the MR increases from $-7 \%$ at $V=0$ to $+21 \%$ at $V=0.6 \mathrm{~V}$, before dropping again to $-1 \%$ at $V=0.8 \mathrm{~V}$. The calculated total currents $I_{P}$ and $I_{\mathrm{AP}}$ as a function of the applied bias $V$ are shown in Fig. 6(b). The currents are distinctly nonlinear, and the junction is nonohmic. The transmissions of the bilayer $\mathrm{C}_{60}$ junction at energies in the interval $E_{F} \pm 0.5 \mathrm{eV}$ are of order $10^{-2}$ [see Figs. 4(a) and 5(a)], which, although much smaller than unity, is still larger than is typical for a tunnel junction. In other words, a bilayer $\mathrm{C}_{60}$ junction is still quite a leaky junction.

Figure 6(c) shows the spin polarization of the current or current polarization $(\mathrm{CP})$. At zero bias, $V=0$, the total current $I_{P}=I^{\uparrow}+I^{\downarrow}$ through a parallel junction is not polarized, i.e., $I^{\uparrow}=I^{\downarrow}$. Upon increasing the bias the minority spin current becomes dominant, $I^{\downarrow}>I^{\uparrow}$, and the total current $I_{P}$ becomes polarized with a minimum of $-35 \%$ at $V=0.3 \mathrm{~V}$. Remarkably, a current polarization of a similar magnitude can be achieved in an AP junction, albeit at a bias that is more than twice as large.

Figure 7 shows the transmission spectra $T_{P}^{\sigma}(E, V)$ at finite bias, calculated self-consistently, for a range of different biases. The factorization model allows one to interpret the trends in these spectra, and in the MR and the CP. According to Eq. (8) one can construct the transmission at finite bias starting by multiplying a pair of $\sqrt{T_{P}^{\sigma}}$ spectra, displaced by $\pm e V / 2$, cf. Fig. 3. At zero bias the $\mathrm{CP}$ is zero, reflecting the

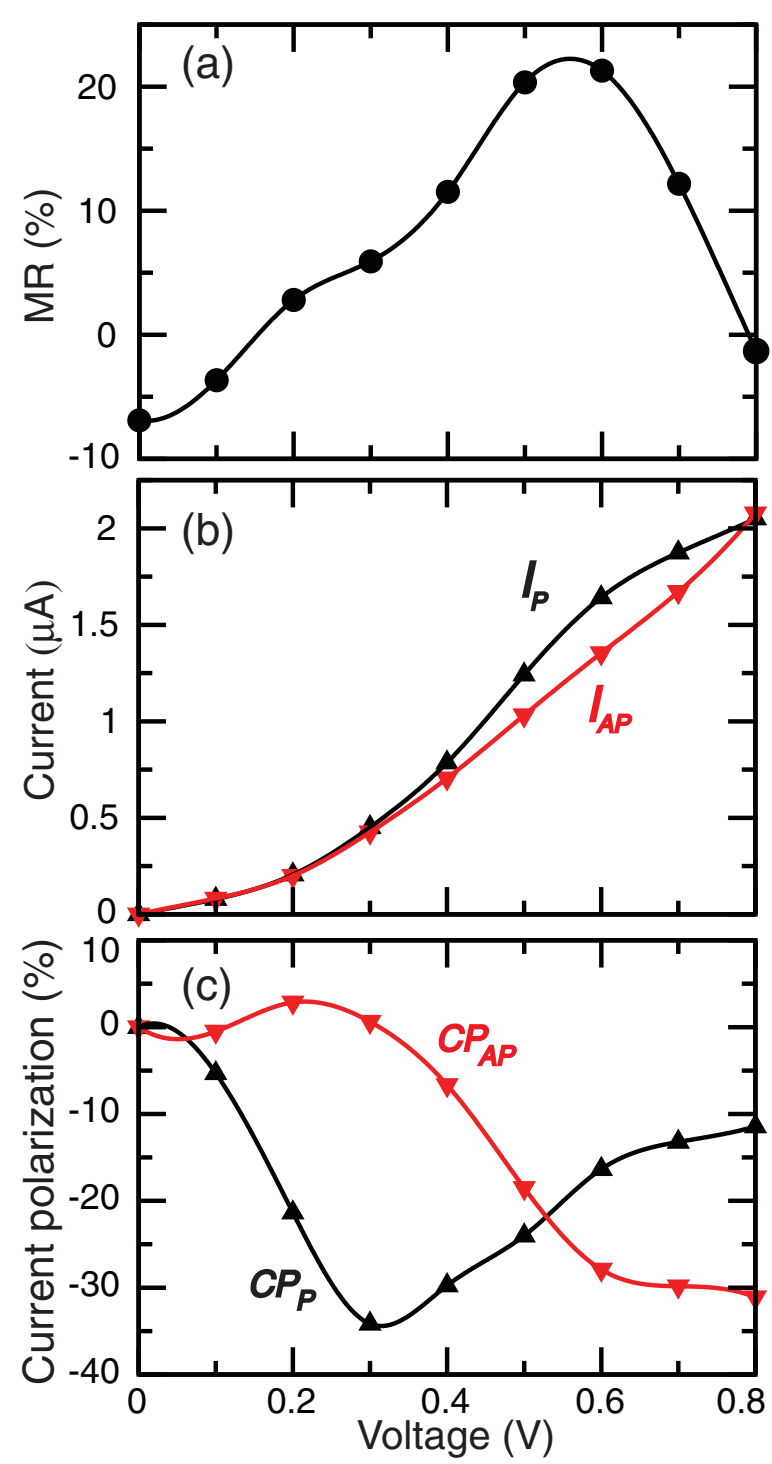

FIG. 6. (Color online) (a) Magnetoresistance $\mathrm{MR}=\left(I_{P}-I_{\mathrm{AP}}\right) /$ $I_{\mathrm{AP}}$ of the $\mathrm{C}_{60}$ bilayer junction as function of bias $V$. (b) Total currents $I_{P}$ (black) and $I_{\mathrm{AP}}$ (red) for the magnetizations of both electrodes parallel, respectively, antiparallel. (c) Current polarization $\mathrm{CP}=\left(I^{\uparrow}-I^{\downarrow}\right) /\left(I^{\uparrow}+I^{\downarrow}\right)$.

fact that $T_{P}^{\uparrow}\left(E_{F}, 0\right)=T_{P}^{\downarrow}\left(E_{F}, 0\right)$. Close to the Fermi level at $E \approx E_{F} \pm 0.2 \mathrm{eV}$, the minority spin transmission $T_{P}^{\downarrow}(E, 0)$ shows two small peaks, Fig. 3(a). Both these peaks are derived from interface states involving the $\mathrm{C}_{60}$ LUMO, as discussed in Appendix B.

Increasing the bias means that these two peaks move towards one another, according to Eq. (8). If one displaces the $T_{P}^{\downarrow}(E, 0)$ spectra by $\pm 0.2 \mathrm{eV}$, these two peaks coincide at the same energy. According to Eq. (8) such a displacement corresponds to a bias of $0.4 \mathrm{~V}$. As the majority spin transmission $T_{P}^{\uparrow}(E, 0)$ does not have peaks in this energy region, it means that at this bias the $\mathrm{CP}$ is negative, which indeed is the case, as can be seen in Fig. 6(c). Upon increasing the bias further, peaks in the majority spin transmission also move into the integration window for the total current. This means that the 


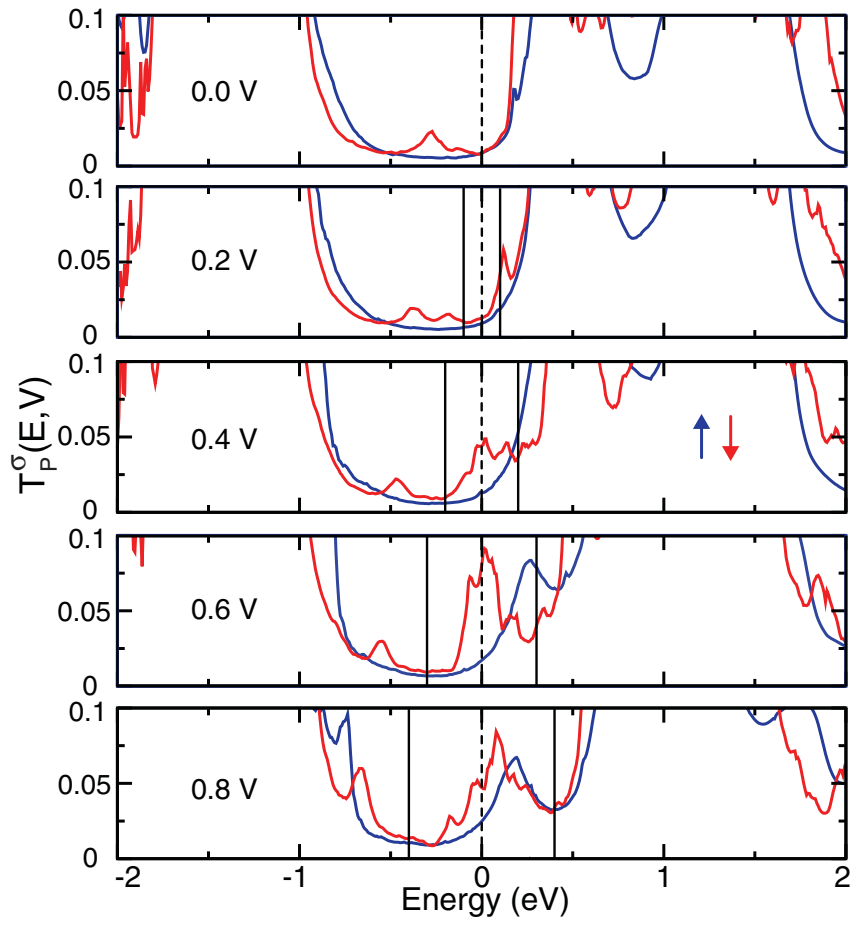

FIG. 7. (Color online) $T_{P}^{\uparrow}(E, V)$ for majority spin (blue) and $T_{P}^{\downarrow}(E, V)$ for minority spin (red) of the $\mathrm{C}_{60}$ bilayer junction as a function of bias, from top to bottom: $V=0.0,0.2,0.4,0.6,0.8 \mathrm{~V}$. The vertical lines enclose the energy interval over which to integrate to obtain the total current according to Eq. (1).

CP decreases with increasing bias, which can also be observed [Fig. 6(c)].

To explain the $\mathrm{CP}$ for the $\mathrm{AP}$ case one must realize that the role of majority and minority spin are now interchanged for one of the electrodes. Within the factorization model, one has to multiply a $\sqrt{T_{P}^{\sigma}}$ curve with a $\sqrt{T_{P}^{-\sigma}}$ curve, displaced by $\pm e V / 2$ (see Fig. 3). The first peak in $T_{P}^{\uparrow}$ is at $E \approx E_{F}+0.5 \mathrm{eV}$. In order to have that coincide with the peak in $T_{P}^{\downarrow}$ at $E \approx E_{F}-0.2 \mathrm{eV}$, it requires a bias $V \approx 0.7 \mathrm{~V}$. Multiplying the $\sqrt{T_{P}^{\sigma}}$ and $\sqrt{T_{P}^{-\sigma}}$ factors then gives a peak in the transmission spectrum $T_{\mathrm{AP}}^{\downarrow}(E, V)$ at $E=0, V=0.7 \mathrm{~V}$. Indeed the self-consistent transmission spectrum $T_{\mathrm{AP}}^{\sigma}$ at finite bias, given in Fig. 8, shows this peak in $T_{\mathrm{AP}}^{\downarrow}$ growing with increasing bias. Therefore, for the AP case one expects to see a zero $\mathrm{CP}$ at low bias, and a decreasing $\mathrm{CP}$ at higher bias, which is indeed the case in Fig. 6(c).

The behavior of the MR in Fig. 6(a) can be interpreted qualitatively along the same lines. The MR is zero at zero bias because the transmission of both spin channels is almost the same. Upon increasing the bias foremost the transmission of the minority spin channel in the $\mathrm{P}$ case increases, cf. Fig. 7, which according to the factorization model originates from shifting the two minority spin peaks at $E \approx E_{F} \pm 0.2 \mathrm{eV}$ closer together [see Fig. 3(a)] as discussed above. There is not such an increase in the AP case, as the roles of majority and minority spin in one of the electrodes are reversed. This means that, upon increasing the bias, $I_{\mathrm{AP}}<I_{P}$ as can be observed in Fig. 6(b). Upon further increase of the bias the transmission of the minority spin channel in the AP case increases, cf. Fig. 8, as

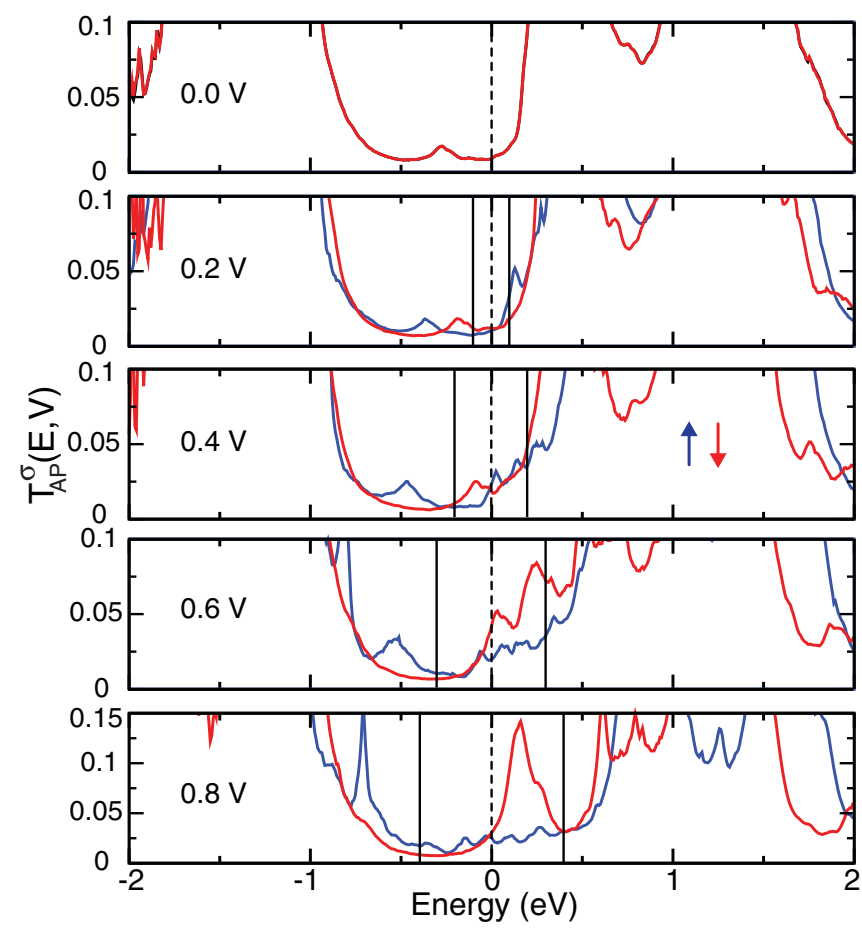

FIG. 8. (Color online) $T_{\mathrm{AP}}^{\uparrow}(E, V)$ for majority spin (blue) and $T_{\mathrm{AP}}^{\downarrow}(E, V)$ for minority spin (red) of the $\mathrm{C}_{60}$ bilayer junction as a function of bias, from top to bottom: $V=0.0,0.2,0.4,0.6,0.8 \mathrm{~V}$. The vertical lines enclose the energy interval over which to integrate to obtain the total current according to Eq. (1).

the minority spin peak at $E \approx E_{F}-0.2 \mathrm{eV}$ starts to approach the majority spin peak at $E \approx E_{F}+0.5 \mathrm{eV}$, as discussed in the previous paragraph. It means that at a higher bias $I_{\mathrm{AP}}$ increases relative to $I_{P}$, and the MR decreases again [see Fig. 6(a)].

The behavior of the $\mathrm{Fe}(001) \mid$ bilayer $\mathrm{C}_{70} \mid \mathrm{Fe}(001)$ junction as a function of bias voltage has been explained in Ref. [47]. It is much simpler than that of the bilayer $\mathrm{C}_{60}$ junction. At zero bias a peak in the transmission for minority spin is found very close to the Fermi level, which results in a substantial CP and MR at zero bias. Upon increasing the bias this peak decreases as in the factorization model the two factors are displaced from one another; cf. Eq. (9). That results in a monotonic decrease of both $|\mathrm{CP}|$ and $|\mathrm{MR}|$ as a function of bias.

The transmission spectra of the molecular trilayer junctions are qualitatively similar to those of their corresponding bilayers (see Figs. 4 and 5). This means that as a function of bias one expects the $\mathrm{CP}$ and the MR of trilayers to behave similarly to their bilayer counterparts. Of course the absolute currents for the trilayer cases will be much lower than for the bilayer cases. The fact that the general behavior of the $\mathrm{CP}$ and the MR does not depend critically on the thickness of the molecular layers illustrates the central role played by the metal-molecule interfaces.

\section{SUMMARY AND CONCLUSIONS}

We calculate the electronic transport from first principles through spin valves composed of bilayers and trilayers of the fullerene molecules $\mathrm{C}_{60}$ and $\mathrm{C}_{70}$, sandwiched between two ferromagnetic Fe electrodes. Despite the similarity of the two 
molecules, they give rise to a markedly different behavior of their spin-dependent currents. $\mathrm{C}_{70}$ bi- and trilayers give large negative current polarizations of -80 to $-90 \%$ at small bias, where the minus sign indicates that the currents are dominated by minority spin. In contrast, the current polarization generated by $\mathrm{C}_{60}$ layers is zero at small bias. Similarly, the magnetoresistance of $\mathrm{C}_{70}$ spin valves at small bias is $70 \%$ to $140 \%$, whereas that of $\mathrm{C}_{60}$ spin valves is only a few percent. As a function of applied bias across the spin valve, the current polarization of $\mathrm{C}_{70}$ junctions increases monotonically toward zero, and the magnetoresistance decreases toward zero. For bilayer $\mathrm{C}_{60}$ spin valves the current polarization goes through a minimum of $-35 \%$ at $V=0.3 \mathrm{~V}$, as a function of applied bias, and the magnetoresistance goes through a maximum of $24 \%$ at $V=0.55 \mathrm{~V}$.

All these trends can be explained using a generalized Jullière or factorization model, which couples the spindependent transport of the junctions to the electronic structure of the molecule-metal interfaces. The favorable properties of $\mathrm{C}_{70}$ junctions can be traced to an interface state in the minority spin, which is derived from the molecular LUMO, and lies very close the Fermi energy. Although a similar state also exists for $\mathrm{C}_{60}$, it lies $\sim 0.2 \mathrm{eV}$ below the Fermi level, which means that it becomes accessible only at a higher bias voltage. The binding of the molecules to the surface plays a decisive role in determining the position of these states with respect to the Fermi level. Increasing the thickness of the molecular layers decreases the absolute value of the currents, but it has a relative small effect on the sizes of the current polarization and of the magnetoresistance, which stresses the pivotal role played by the molecule-metal interfaces.

\section{ACKNOWLEDGMENTS}

We thank Michel de Jong and Zhe Yuan for useful discussions. Computational resources were provided through the Physical Sciences division of the Netherlands Organization for Scientific Research (NWO-EW) and by TUBITAK ULAKBIM, High Performance and Grid Computing Center (TR-Grid e-Infrastructure).

\section{APPENDIX A: PARTITIONING}

To calculate the transmission, Eq. (2), one needs the block of the Green's function matrix $\mathbf{G}_{R L}$ connecting the right and left leads via the quantum conductor, where we omit the spin index for the moment to simplify the notation. We partition the system into a left and a right part,

$$
\left(\begin{array}{ll}
\mathbf{G}_{L L} & \mathbf{G}_{L R} \\
\mathbf{G}_{R L} & \mathbf{G}_{R R}
\end{array}\right)\left(\begin{array}{cc}
E \mathbf{I}_{L}-\mathbf{H}_{L L} & -\mathbf{H}_{L R} \\
-\mathbf{H}_{R L} & E \mathbf{I}_{R}-\mathbf{H}_{R R}
\end{array}\right)=\left(\begin{array}{cc}
\mathbf{I}_{L} & 0 \\
0 & \mathbf{I}_{R}
\end{array}\right),
$$

where the diagonal blocks $\mathbf{H}_{L L}$ and $\mathbf{H}_{R R}$ of the Hamiltonian matrix represent the semi-infinite left and right parts, and the off-diagonal blocks $\mathbf{H}_{R L}=\left(\mathbf{H}_{L R}\right)^{\dagger}$ represent the coupling between the right and left parts.

Formally solving Eq. (A1) then gives for the off-diagonal block of the Green's function matrix,

$$
\mathbf{G}_{R L}=\mathbf{g}_{R} \mathbf{H}_{R L}\left(\mathbf{I}_{L}-\mathbf{g}_{L} \mathbf{H}_{L R} \mathbf{g}_{R} \mathbf{H}_{R L}\right)^{-1} \mathbf{g}_{L},
$$

with

$$
\mathbf{g}_{R}=\left(E \mathbf{I}_{R}-\mathbf{H}_{R R}\right)^{-1} ; \mathbf{g}_{L}=\left(E \mathbf{I}_{L}-\mathbf{H}_{L L}\right)^{-1},
$$

the Green's function matrices of the uncoupled right and left parts. Expression (A2) can be used to rewrite Eq. (2). Moreover, it is easy to show that $\mathbf{g}_{R(L)}^{a} \boldsymbol{\Gamma}_{R(L)} \mathbf{g}_{R(L)}^{r}=2 \pi \mathbf{n}_{R(L)}$, where $\mathbf{n}_{R(L)}=-\pi^{-1} \operatorname{Img}_{R(L)}^{r}$ is the spectral density matrix of the right (left) part [48,52]. Equation (2) then becomes

$$
\begin{aligned}
T= & 4 \pi^{2} \operatorname{Tr}\left[\mathbf{n}_{R} \mathbf{H}_{R L}\left(\mathbf{I}_{L}-\mathbf{g}_{L}^{r} \mathbf{H}_{L R} \mathbf{g}_{R}^{r} \mathbf{H}_{R L}\right)^{-1},\right. \\
& \left.\times \mathbf{n}_{L} \mathbf{H}_{L R}\left(\mathbf{I}_{R}-\mathbf{g}_{R}^{a} \mathbf{H}_{R L} \mathbf{g}_{L}^{a} \mathbf{H}_{L R}\right)^{-1}\right] .
\end{aligned}
$$

A similar expression has been derived in Ref. [64] to model scanning tunneling microscopy. It can also be derived from the (linear response) Kubo formalism, as in Refs. [65,66]. The expression is, however, also valid outside the linear response regime, cf. Eq. (1), provided the density, Hamiltonian, and Green's function matrices are calculated self-consistently [50].

The terms $\left(\mathbf{I}_{L}-\cdots\right)^{-1}$ and $\left(\mathbf{I}_{R}-\cdots\right)^{-1}$ in Eq. (A4) incorporate the effects of (multiple) reflections between the left and right parts. Neglecting these, i.e., replacing these terms by $\mathbf{I}_{L}$ and $\mathbf{I}_{R}$, respectively, then gives

$$
T=4 \pi^{2} \operatorname{Tr}\left[\mathbf{n}_{R} \mathbf{H}_{R L} \mathbf{n}_{L} \mathbf{H}_{L R}\right] .
$$

One expects this approximation to be accurate in the tunneling regime. Reintroducing the spin index $\sigma$, and choosing representations where the density matrices are diagonal, $\left(\mathbf{n}_{R}^{\sigma}\right)_{i j}=$ $\delta_{i j} n_{R i}^{\sigma},\left(\mathbf{n}_{L}^{\sigma}\right)_{i j}=\delta_{i j} n_{L j}^{\sigma}$, then gives Eq. (3).

\section{APPENDIX B: FULLERENE|Fe(001) INTERFACES}

Transport calculations of the type used here require a real space representation of the Hamiltonian, for which basis sets comprising localized atomic orbitals are ideally suited, as they are used in TRANSIESTA [50,61]. However, that program uses norm-conserving pseudopotentials (NCPPs) [62], which can lead to an overestimation of magnetic effects (exchange splitting, magnetic moments). Therefore, we benchmark SIESTA NCPP results against results obtained with (allelectron) projector-augmented waves (PAWs) [56,57], as used in VASP [58,59].

We optimize all structures with VASP, using the PBE functional [60], and the parameter settings given in Sec. III. The optimized lattice constant of bulk Fe is $2.83 \AA$, which is in good agreement with the experimental values of $2.87 \AA$ [67]. The magnetic moments per atom of bulk Fe are $2.20 \mu_{B}$ (VASP) and $2.25 \mu_{B}$ (SIESTA), respectively, which both are in good agreement with the experimental value of $2.22 \mu_{B}$ [67]. The difference between the magnetic moments calculated with VASP and SIESTA can be traced to the use of NCPPs in SIESTA, versus (all-electron) PAWs in VASP. The former gives a larger exchange splitting (see Fig. 9), which gives a larger magnetic moment. VASP calculations with NCPPs give a similar exchange splitting as SIESTA [68], so the use of different basis sets in VASP and SIESTA, i.e., plane waves versus localized atomic orbitals, is of less importance.

The difference in calculated magnetic moments between PAWs and NCPPs persists for the $\mathrm{Fe}(001)$ surface. Figure 9 gives the magnetic moments as a function of layer for a $\mathrm{Fe}(001)$ slab. The magnetic moment of a surface atom is $\sim 3 \mu_{B}$ and 

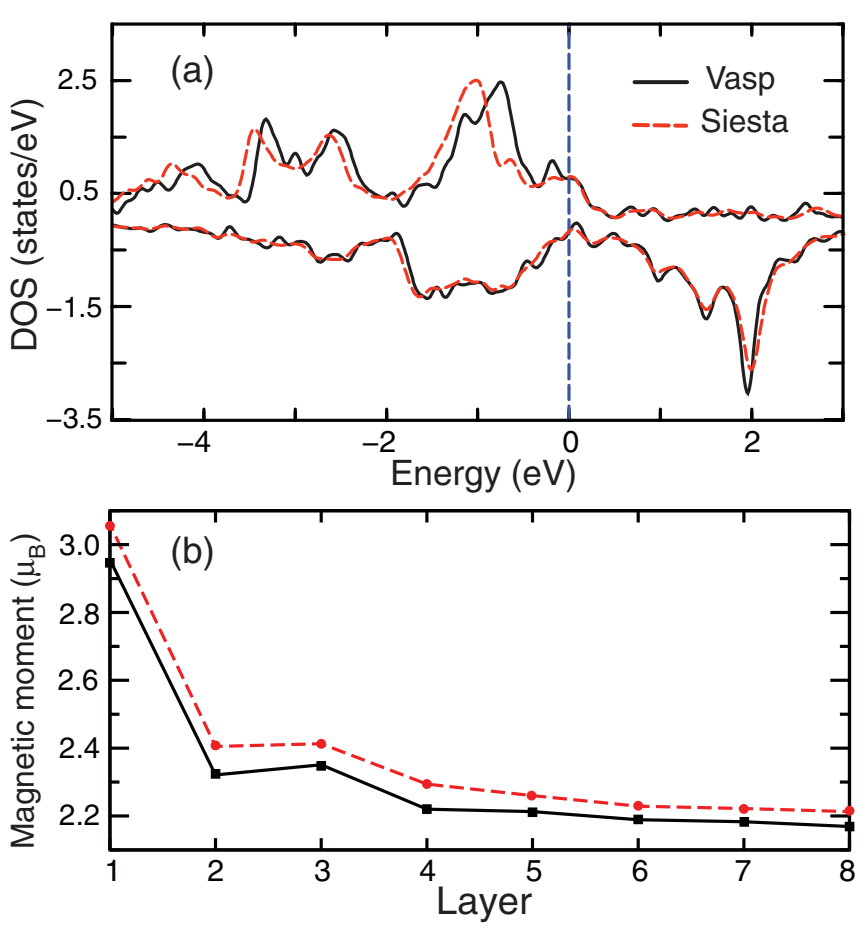

FIG. 9. (Color online) (a) Density of states of bulk bcc Fe, calculated with PAW (black) and NCPP (red). The NCPP gives an exchange splitting that is $\sim 0.2 \mathrm{eV}$ larger, which results in a $\sim 0.05 \mu_{B}$ larger magnetic moment. (b) Magnetic moments (in $\mu_{B} /$ atom) of the $\mathrm{Fe}(001)$ surface as function of layer position (1 indicates the surface layer) calculated with PAW (black) and NCPP (red).

the difference between the VASP and the SIESTA results is about $3 \%$. It is well known that $d$-band narrowing for surface atoms enhances the exchange splitting, resulting in a larger magnetic moment for the surface atoms as compared to bulk [69].

To model the adsorption of $\mathrm{C}_{60}$ and $\mathrm{C}_{70}$ molecules we use a slab of seven atomic layers for the $\mathrm{Fe}(001)$ substrate with a layer of molecules absorbed on one side of the slab, and $15 \AA$ of vacuum thickness. The molecules and the uppermost three $\mathrm{Fe}$ atomic layers are allowed to relax. A dipole correction is applied to prevent spurious interactions between repeated images of the slab [70].

From a number of possible adsorption geometries, we have identified the structure of adsorbed $\mathrm{C}_{60}$ molecules shown in Fig. 10(a) as the most stable. The edge shared by two hexagons (a 6:6 bond) is on top of a surface Fe atom, and the $\mathrm{C}_{60}$ molecule is tilted such that one of the hexagons is more parallel to the surface. There are several short $\mathrm{Fe}-\mathrm{C}$ bonds in the range 2.0-2.5 $\AA$, which is an indication of chemisorption, as is confirmed by the binding energy (see Table II). The C-C bond lengths within these two hexagons are between 1.46 and $1.52 \AA$, i.e., somewhat larger than the $6: 6$ and 5:6 bond lengths of 1.40 and $1.46 \AA$ in an unperturbed $\mathrm{C}_{60}$ molecule. Judging from the changes in bond lengths, the interaction with the $\mathrm{Fe}(001)$ surface seems to break the conjugation in these two hexagons somewhat. The $\mathrm{C}-\mathrm{C}$ bonds in the other hexagons and pentagons are hardly perturbed by the adsorption. Upon adsorption of a monolayer of $\mathrm{C}_{60}$ molecules, the work function of $\mathrm{Fe}(001)$ increases by $0.94 \mathrm{eV}$. The increase indicates that the $\mathrm{C}_{60}$ molecule acts as an electron acceptor which is consistent (a)
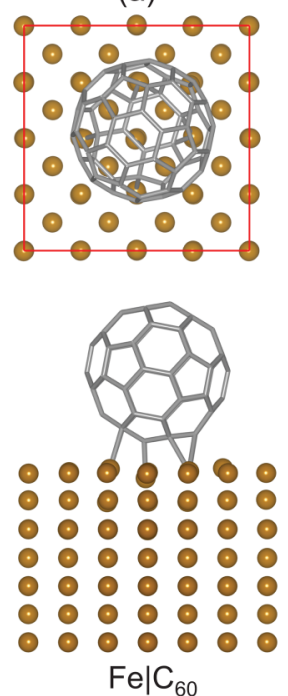

(b)
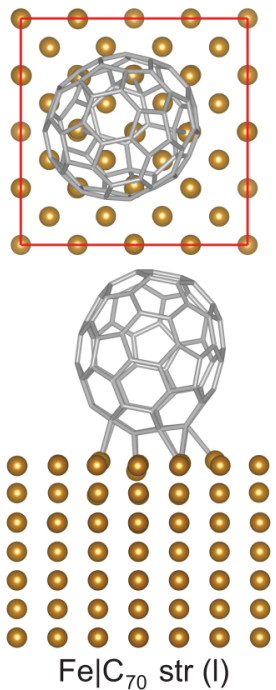

(c)
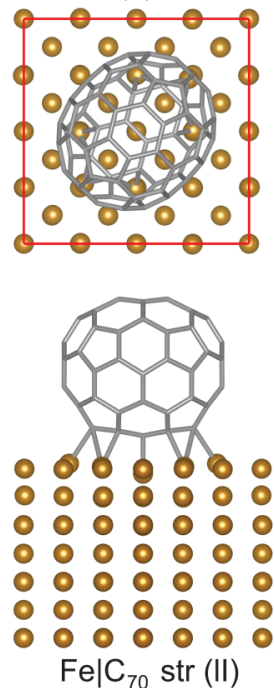

FIG. 10. (Color online) (a) Top and side views of the most stable adsorption geometry of $\mathrm{C}_{60}$ on $\mathrm{Fe}(001)$; $\mathrm{Fe}-\mathrm{C}$ distances below $2.5 \AA$ are indicated specifically. $\mathrm{C}_{70}$ on $\mathrm{Fe}(001)$ in structure (I) (b) and structure (II) (c).

with the high electron affinity of $4.5 \mathrm{eV}$ of this molecule. The interaction with the ferromagnetic Fe surface induces a small magnetic moment of $0.22 \mu_{B}$ on the $\mathrm{C}_{60}$ molecule [38].

One can form a bonding geometry of the $\mathrm{C}_{70}$ molecule to the $\mathrm{Fe}(001)$ surface that is very similar to that of $\mathrm{C}_{60}$; see Fig. 10(b). This structure (I) has the edge shared by two hexagons on top of a surface Fe atom, and the $\mathrm{C}_{70}$ molecule is tilted such that one of the hexagons is more parallel to the surface. $\mathrm{C}_{70}$ in this structure has similar properties as $\mathrm{C}_{60}$ (see Table II), but it is not the lowest energy structure. We find that in the most stable adsorption geometry, structure (II), the long axis of the $\mathrm{C}_{70}$ molecule is parallel to the surface [see Fig. 10(c)]. Like in structure (I), in structure (II) the edge shared by two hexagons is on top of a surface Fe atom, but unlike structure (I) the two hexagons in $\mathrm{C}_{70}$ have a symmetric tilt with respect to the surface. Again, there are several short $\mathrm{Fe}-\mathrm{C}$ bonds in the range $2.0-2.3 \AA$. The $\mathrm{C}-\mathrm{C}$ distances in the two hexagons involved in the adsorption are in the range $1.45-1.50 \AA$, again somewhat larger than the $1.39-1.47$ and 1.44-1.45 $\AA$ of the $6: 6$ and 5:6 bonds, respectively, of

TABLE II. Binding energies $E_{b}$ of $\mathrm{C}_{60}$ and $\mathrm{C}_{70}$ molecules on $\mathrm{Fe}(001)$ of the structures shown in Fig. 10 [total energies of unperturbed $\mathrm{Fe}(001)$ and isolated fullerene minus total energy of fullerene adsorbed on $\mathrm{Fe}(001)]$; work function $W$ of $\mathrm{Fe}(001)$ covered by a monolayer of fullerenes; magnetic moment $\mu$ induced on the fullerene molecules; spin polarization SP of the density of states at the Fermi level, projected on the fullerene molecules.

\begin{tabular}{lcccc}
\hline \hline Structure & $E_{b}(\mathrm{eV})$ & $W(\mathrm{eV})^{\mathrm{a}}$ & $\mu\left(\mu_{B}\right)$ & $\mathrm{SP}(\%)$ \\
\hline $\mathrm{C}_{60}$ & 2.94 & 4.81 & 0.22 & 0 \\
$\mathrm{C}_{70}(\mathrm{I})$ & 2.79 & 4.67 & 0.22 & 0 \\
$\mathrm{C}_{70}(\mathrm{II})$ & 2.99 & 4.79 & 0.22 & 40 \\
\hline \hline
\end{tabular}

${ }^{\mathrm{a}}$ Calculated work function of clean $\mathrm{Fe}(001)$ is $3.87 \mathrm{eV}$. 

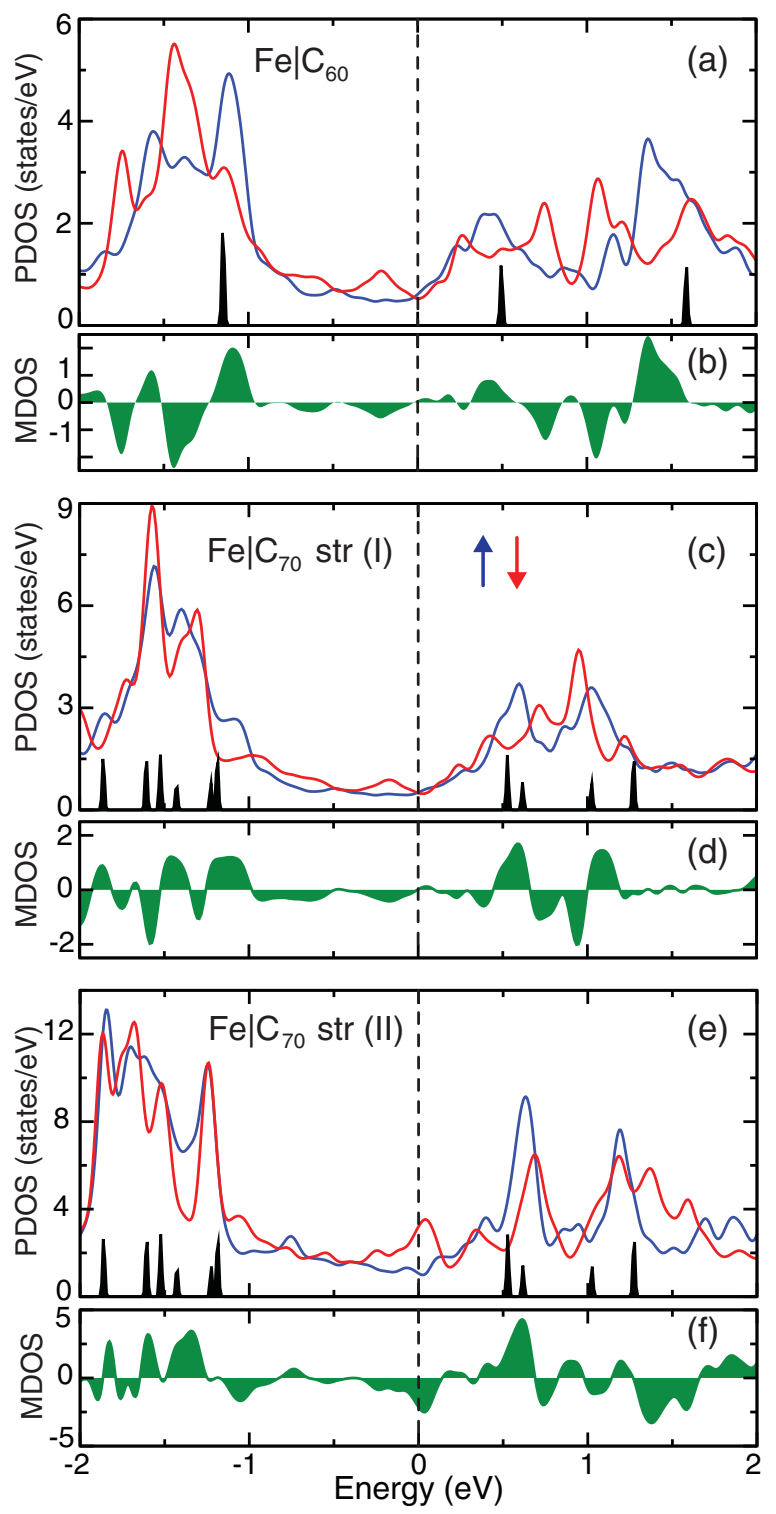

FIG. 11. (Color online) (a) Projected density of states PDOS $n^{\uparrow}$ of majority (blue) and $n^{\downarrow}$ of minority (red) spin states of the $\mathrm{Fe}(001) \mid \mathrm{C}_{60}$ interface, summed over all carbon atoms. Gaussian smearing with a smearing parameter of $0.05 \mathrm{eV}$ is applied. The black lines give the energy levels of the isolated $\mathrm{C}_{60}$ molecule. (b) Magnetization density of states MDOS $\Delta n=n^{\uparrow}-n^{\downarrow}$; (c) and (d) PDOS and MDOS of $\mathrm{Fe}(001) \mid \mathrm{C}_{70}$ in structure (I), and (e) and (f) in structure (II).

the isolated $\mathrm{C}_{70}$ molecule, whereas the bond lengths in the rest of the molecule are hardly changed. As structure (II) is $0.2 \mathrm{eV} / \mathrm{C}_{70}$ molecule more stable than structure (I), we have used structure (II) in all our transport calculations.

The magnetic moments induced on the $\mathrm{C}_{60}$ and $\mathrm{C}_{70}$ molecules are similar, and do not depend very much on the details of the structure (see Table II). Of course only the spin polarization of the states around the Fermi level is important when studying electron transport, and not the overall polarization or magnetic moment. Figure 11(a) gives the projected density of states (PDOS) of the $\mathrm{Fe}(001) \mid \mathrm{C}_{60}$ interface, summed over all the carbon atoms. For comparison the Kohn-Sham levels of the isolated $\mathrm{C}_{60}$ molecule are also given, which can be aligned with the interface DOS using the lowest $\sigma$ states of the $\mathrm{C}_{60}$ molecule. The latter do not participate in the bonding to the surface, and are therefore not perturbed.

The $\pi$ states of the molecule, however, hybridize with states from the substrate. These molecular states can still be identified from the peaks in the PDOS, but the peaks are significantly broadened and shifted, compared to the isolated molecule. The isocahedral symmetry $I_{h}$ of the $\mathrm{C}_{60}$ molecule is broken by adsorption on the Fe surface, which lifts degeneracies and splits up the peaks of the adsorbed $\mathrm{C}_{60}$ molecule. In addition, the $\mathrm{Fe}(001)$ substrate interacts differently with the molecule for different spin states. The $\mathrm{Fe}(001)$ surface has prominent surface resonances in the minority spin channel for energies close to the Fermi level [71]. The corresponding wave functions have a relatively long decay length, and one can expect these states to interact strongly with adsorbants. Indeed the minority spin states in the PDOS show a stronger perturbation with respect to the molecular $\pi$ states than the majority spin states, in particular for energies around the Fermi level.

Comparison to the states of the isolated $\mathrm{C}_{60}$ molecule allows one to label the corresponding peaks in PDOS of the adsorbed molecule. Of course, adsorption broadens the peaks, and sometimes splits them. For instance, the fivefold degeneracy of the molecular HOMO is clearly lifted. In the minority spin states the LUMO, as well as the LUMO + 1, which are both threefold degenerate in the isolated molecule, are split up. One of the states derived from the LUMO results in a peak in the minority spin DOS at $E_{F}-0.2 \mathrm{eV}$, whereas other LUMO derived peaks appear above $E_{F}+0.2 \mathrm{eV}$. It indicates that adsorption results in a net transfer of electrons to the $\mathrm{C}_{60}$ molecule, which is consistent with an increase of the work function (see Table II). At the Fermi level, $E=E_{F}$, the PDOSs of majority and minority spins are nearly equal, which implies that the spin polarization $\Delta n=n^{\uparrow}-n^{\downarrow} \approx 0$ [see Fig. 11(b)].

Figure 11(c) gives the PDOS of the $\mathrm{Fe}(001) \mid \mathrm{C}_{70}$ interface, with the $\mathrm{C}_{70}$ in structure (I). Again for comparison the Kohn-Sham levels of the isolated $\mathrm{C}_{70}$ molecule are also given. The level spectrum of $\mathrm{C}_{70}$ is somewhat denser than that of $\mathrm{C}_{60}$, as the molecule is slightly larger and less symmetric. Nevertheless, qualitatively the PDOS is remarkably similar to that of $\mathrm{C}_{60}$. Specifically, also for $\mathrm{C}_{70}$ in structure (I) one of the LUMO-derived states gives a peak in the minority spin at $E_{F}-0.2 \mathrm{eV}$, and other LUMO-derived peaks appear above $E_{F}+0.2 \mathrm{eV}$. (a)

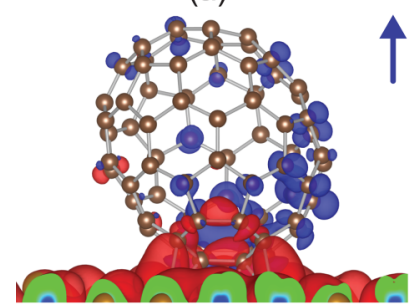

$\mathrm{Fe} \mid \mathrm{C}_{70} \operatorname{str}(\mathrm{I})$ (b)

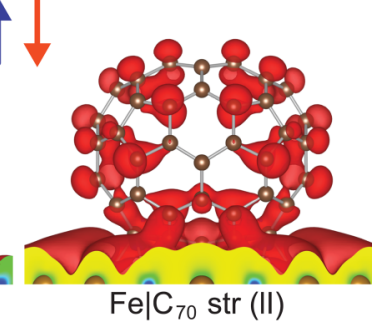

FIG. 12. (Color online) (a) and (b) Spin polarization of the LDOS at the $\mathrm{Fe}(001) \mid \mathrm{C}_{70}$ interface in structure I, respectively, structure II, integrated over an energy interval $\left[E_{F}-0.01, E_{F}+0.01\right] \mathrm{eV}$. 
Figure 11(e) gives the PDOS of the $\mathrm{Fe}(001) \mid \mathrm{C}_{70}$ interface, with the $\mathrm{C}_{70}$ in structure (II). Although qualitatively this PDOS is similar to that of $\mathrm{C}_{70}$ in structure (I), there are nevertheless important differences, specifically for energies around the Fermi level. For $\mathrm{C}_{70}$ in structure (II) a hybrid state with $\mathrm{C}_{70}$ LUMO character gives a prominent peak in the minority spin channel, that is, at the Fermi level, instead of $0.2 \mathrm{eV}$ below $E_{F}$, as is the case for $\mathrm{C}_{70}$ in structure (I) and for $\mathrm{C}_{60}$. This means that the spin polarization at $E=E_{F}$, $\mathrm{SP}=\left(n^{\uparrow}-n^{\downarrow}\right) /\left(n^{\uparrow}+n^{\downarrow}\right) \approx 40 \%$ for $\mathrm{C}_{70}$ in structure (II), which also implies that the MR in this structure is markedly different, as discussed in Sec. IV.
The difference between $\mathrm{C}_{60}$ and $\mathrm{C}_{70}$ in structure (I) on the one hand, and $\mathrm{C}_{70}$ in structure (II) on the other, is also reflected in the wave function at the the Fermi level. Figure 12 shows the spin polarization in the local density of states (LDOS), integrated over an energy interval of $\pm 0.01 \mathrm{eV}$ around the Fermi level. The LDOS of $\mathrm{C}_{70}$ in structure (II) clearly shows shows a hybrid state with clear contributions both from the $\mathrm{C}_{70}$ molecule and the $\mathrm{Fe}(001)$ substrate, which is delocalized over the whole molecule, and has a clear minority spin character. In contrast, the LDOS of $\mathrm{C}_{70}$ in structure (I) shows a hybrid state that covers only part of the molecule, and has a mixed majority/minority spin character.
[1] A. Fert, Rev. Mod. Phys. 80, 1517 (2008).

[2] I. Zutic, J. Fabian, and S. D. Sarma, Rev. Mod. Phys. 76, 323 (2004).

[3] N. Tombros, C. Jozsa, M. Popinciuc, H. T. Jonkman, and B. J. van Wees, Nature (London) 448, 571 (2007).

[4] V. A. Dediu, L. E. Hueso, I. Bergenti, and C. Taliani, Nature Mater. 8, 707 (2009).

[5] S. Schmaus, A. Bagrets, Y. Nahas, T. K. Yamada, A. Bork, M. Bowen, E. Beaurepaire, F. Evers, and W. Wulfhekel, Nature Nanotechnol. 6, 185 (2011).

[6] S. L. Kawahara, J. Lagoute, V. Repain, C. Chacon, Y. Girard, S. Rousset, A. Smogunov, and C. Barreteau, Nano Lett. 12, 4558 (2012).

[7] T. Miyamachi, M. Gruber, V. Davesne, M. Bowen, S. Boukari, L. Joly, F. Scheurer, G. Rogez, T. K. Yamada, P. Ohresser, E. Beaurepaire, and W. Wulfhekel, Nature Commun. 3, 938 (2012).

[8] K. Yoshida, I. Hamada, S. Sakata, A. Umeno, M. Tsukada, and K. Hirakawa, Nano Lett. 13, 481 (2013).

[9] Z. H. Xiong, D. Wu, and V. Vardeny, Nature (London) 427, 821 (2004).

[10] T. S. Santos, J. S. Lee, P. Migdal, I. C. Lekshmi, B. Satpati, and J. S. Moodera, Phys. Rev. Lett. 98, 016601 (2007).

[11] V. Dediu, L. E. Hueso, I. Bergenti, A. Riminucci, F. Borgatti, P. Graziosi, C. Newby, F. Casoli, M. P. DeJong, C. Taliani, and Y. Zhan, Phys. Rev. B 78, 115203 (2008).

[12] J. J. H. M. Schoonus, P. G. E. Lumens, W. Wagemans, J. T. Kohlhepp, P. A. Bobbert, H. J. M. Swagten, and B. Koopmans, Phys. Rev. Lett. 103, 146601 (2009).

[13] D. Sun, L. Yin, C. Sun, H. Guo, Z. Gai, X.-G. Zhang, T. Z. Ward, Z. Cheng, and J. Shen, Phys. Rev. Lett. 104, 236602 (2010).

[14] C. Barraud, P. Seneor, R. Mattana, S. Fusil, K. Bouzehouane, C. Deranlot, P. Graziosi, L. Hueso, I. Bergenti, V. A. Dediu, F. Petroff, and A. Fert, Nat. Phys. 6, 615 (2010).

[15] L. Schulz, L. Nuccio, M. Willis, P. Desai, P. Shakya, T. Kreouzis, V. K. Malik, C. Bernhard, F. L. Pratt, N. A. Morley, A. Suter, G. J. Nieuwenhuys, T. Prokscha, E. Morenzoni, W. P. Gillin, and A. J. Drew, Nature Mater. 10, 39 (2011).

[16] M. Gobbi, F. Golmar, R. Llopis, F. Casanova, and L. E. Hueso, Adv. Mater. 23, 1609 (2011).

[17] T. L. A. Tran, T. Q. Le, J. G. M. Sanderink, W. G. van der Wiel, and M. P. de Jong, Adv. Func. Mater. 22, 1180 (2012).

[18] X. Zhang, S. Mizukami, T. Kubota, Q. Ma, M. Oogane, H. Naganuma, Y. Ando, and T. Miyazaki, Nature Commun. 4, 1392 (2013).
[19] X. Zhang, S. Mizukami, Q. Ma, T. Kubota, M. Oogane, H. Naganuma, Y. Ando, and T. Miyazaki, J. Appl. Phys. 115, 172608 (2014).

[20] F. Li, T. Li, F. Chen, and F. Zhang, Org. Elec. 15, 1657 (2014).

[21] K. V. Raman, A. M. Kamerbeek, A. Mukherjee, N. Atodiresei, T. K. Sen, P. Lazić, V. Casiuc, D. Stalke, S. K. Mandal, and S. Blügel, Nature (London) 493, 509 (2013).

[22] S. Sanvito, Nat. Phys. 6, 562 (2010).

[23] M. Cinchetti, K. Heimerand, J.-P. Wüstenberg, O. Andreyev, M. Bauer, S. Lach, C. Ziegler, Y. Gao, and M. Aeschlimann, Nature Mater. 8, 115 (2008).

[24] Y.-L. Chan, Y.-J. Hung, C.-H. Wang, Y.-C. Lin, C.-Y. Chiu, Y.-L. Lai, H.-T. Chang, C.-H. Lee, Y. J. Hsu, and D. H. Wei, Phys. Rev. Lett. 104, 177204 (2010).

[25] S. Lach, A. Altenhof, K. Tarafder, F. Schmitt, M. E. Ali, M. Vogel, J. Sauther, P. M. Oppeneer, and C. Ziegler, Adv. Funct. Mater. 22, 989 (2012).

[26] S. Steil, N. Großmann, M. Laux, A. Ruffing, D. Steil, M. Wiesenmayer, S. Mathias, O. L. A. Monti, M. Cinchetti, and M. Aeschlimann, Nat. Phys. 9, 242 (2013).

[27] V. A. Dediu, Nat. Phys. 9, 210 (2013).

[28] A. Droghetti, S. Steil, N. Großmann, N. Haag, H. Zhang, M. Willis, W. P. Gillin, A. J. Drew, M. Aeschlimann, S. Sanvito, and M. Cinchetti, Phys. Rev. B 89, 094412 (2014).

[29] S. Shi, Z. Sun, A. Bedoya-Pinto, P. Graziosi, X. Li, X. Liu, L. Hueso, V. A. Dediu, Y. Luo, and M. Fahlman, Adv. Funct. Mater. 24, 4812 (2014).

[30] G. Giovannetti, P. A. Khomyakov, G. Brocks, V. M. Karpan, J. van den Brink, and P. J. Kelly, Phys. Rev. Lett. 101, 026803 (2008).

[31] P. C. Rusu, G. Giovannetti, C. Weijtens, R. Coehoorn, and G. Brocks, Phys. Rev. B 81, 125403 (2010).

[32] M. Bokdam, D. Çakır, and G. Brocks, Appl. Phys. Lett. 98, 113303 (2011)

[33] N. Atodiresei, J. Brede, P. Lazić, V. Caciuc, G. Hoffmann, R. Wiesendanger, and S. Blügel, Phys. Rev. Lett. 105, 066601 (2010).

[34] J. Brede, N. Atodiresei, S. Kuck, P. Lazić, V. Caciuc, Y. Morikawa, G. Hoffmann, S. Blügel, and R. Wiesendanger, Phys. Rev. Lett. 105, 047204 (2010).

[35] S. Javaid, M. Bowen, S. Boukari, L. Joly, J.-B. Beaufrand, X. Chen, Y. J. Dappe, F. Scheurer, J.-P. Kappler, J. Arabski, W. Wulfhekel, M. Alouani, and E. Beaurepaire, Phys. Rev. Lett. 105, 077201 (2010). 
[36] T. L. A. Tran, P. K. J. Wong, M. P. de Jong, W. G. van der Wiel, Y. Q. Zhan, and M. Fahlman, Appl. Phys. Lett. 98, 222505 (2011).

[37] J. Schwöbel, Y. Fu, J. Brede, A. Dilullo, G. Hoffmann, S. Klyatskaya, M. Ruben, and R. Wiesendanger, Nature Comm. 3, 953 (2012).

[38] T. L. A. Tran, D. Çakır, P. K. J. Wong, A. B. Preobrajenski, G. Brocks, W. G. van der Wiel, and M. P. de Jong, ACS Appl. Mater. Interfaces 5, 837 (2013).

[39] F. Djeghloul, F. Ibrahim, M. Cantoni, M. Bowen, L. Joly, S. Boukari, P. Ohresser, F. Bertran, P. L. Fevre, P. Thakur, F. Scheurer, T. Miyamachi, R. Mattana, P. Seneor, A. Jaafar, C. Rinaldi, S. Javaid, J. Arabski, J.-P. Kappler, W. Wulfhekel, N. B. Brookes, R. Bertacco, A. Taleb-Ibrahimi, M. Alouani, E. Beaurepaire, and W. Weber, Sci. Rep. 3, 1272 (2013).

[40] A. R. Rocha, V. M. Garcia-Suarez, S. Bailey, C. Lambert, J. Ferrer, and S. Sanvito, Nature Mater. 4, 335 (2005).

[41] Z. Ning, Y. Zhu, J. Wang, and H. Guo, Phys. Rev. Lett. 100, 056803 (2008).

[42] M. Koleini and M. Brandbyge, Beilstein J. Nanotechnol. 3, 589 (2012).

[43] S. H. Liang, D. P. Liu, L. L. Tao, X. F. Han, and H. Guo, Phys. Rev. B 86, 224419 (2012).

[44] A. Bagrets, S. Schmaus, A. Jaafar, D. Kramczynski, T. K. Yamada, M. Alouani, W. Wulfhekel, and F. Evers, Nano Lett. 12, 5131 (2012).

[45] V. M. Karpan, G. Giovannetti, P. A. Khomyakov, M. Talanana, A. A. Starikov, M. Zwierzycki, J. van den Brink, G. Brocks, and P. J. Kelly, Phys. Rev. Lett. 99, 176602 (2007).

[46] V. M. Karpan, P. A. Khomyakov, A. A. Starikov, G. Giovannetti, M. Zwierzycki, M. Talanana, G. Brocks, J. van den Brink, and P. J. Kelly, Phys. Rev. B 78, 195419 (2008).

[47] D. Çakır, D. M. Otálvaro, and G. Brocks, Phys. Rev. B 89, 115407 (2014).

[48] S. Datta, Electronic Transport in Mesoscopic Systems (Cambridge University Press, Cambridge, 1995).

[49] C. Caroli, R. Combescot, P. Nozières, and D. Saint-James, J. Phys. C: Sol. State Phys. 4, 916 (1971).
[50] M. Brandbyge, J.-L. Mozos, P. Ordejón, J. Taylor, and K. Stokbro, Phys. Rev. B 65, 165401 (2002).

[51] P. A. Khomyakov, G. Brocks, V. M. Karpan, M. Zwierzycki, and P. J. Kelly, Phys. Rev. B 72, 035450 (2005).

[52] G. D. Mahan, Many-Particle Physics, 2nd ed. (Plenum Press, New York/London, 1990).

[53] M. Jullière, Phys. Lett. A 54, 225 (1975).

[54] P. A. Heiney, J. E. Fischer, A. R. McGhie, W. J. Romanow, A. M. Denenstein, J. P. McCauley Jr., A. B. Smith III, and D. E. Cox, Phys. Rev. Lett. 66, 2911 (1991).

[55] S. van Smaalen, V. Petricek, J. L. de Boer, M. Dusek, M. A. Verheijen, and G. Meijer, Chem. Phys. Lett. 223, 323 (1994).

[56] P. E. Blöchl, Phys. Rev. B 50, 17953 (1994).

[57] G. Kresse and D. Joubert, Phys. Rev. B 59, 1758 (1999).

[58] G. Kresse and J. Hafner, Phys. Rev. B 47, 558 (1993).

[59] G. Kresse and J. Furthmüller, Phys. Rev. B 54, 11169 (1996).

[60] J. P. Perdew, K. Burke, and M. Ernzerhof, Phys. Rev. Lett. 77, 3865 (1996).

[61] J. M. Soler, E. Artacho, J. D. Gale, A. García, J. Junquera, P. Ordejón, and D. Sánchez-Portal, J. Phys.: Condens. Matter 14, 2745 (2002).

[62] N. Troullier and J. L. Martins, Phys. Rev. B 43, 1993 (1991).

[63] The transmissions of majority and minority spins are the same in the $\mathrm{AP}$ configuration at zero bias, $T_{\mathrm{AP}}^{\uparrow}(E, 0)=T_{\mathrm{AP}}^{\downarrow}(E, 0)$.

[64] N. Mingo, L. Jurczyszyn, F. J. Garcia-Vidal, R. Saiz-Pardo, P. L. de Andres, F. Flores, S. Y. Wu, and W. More, Phys. Rev. B 54, 2225 (1996).

[65] J. Mathon, Phys. Rev. B 56, 11810 (1997).

[66] J. Mathon and A. Umerski, Phys. Rev. B 60, 1117 (1999).

[67] C. Kittel, Introduction to Solid State Physics, 7th ed. (Wiley, New York, 1986).

[68] G. A. de Wijs (private communication).

[69] J. Izquierdo, A. Vega, L. C. Balbas, D. Sanchez-Portal, J. Junquera, E. Artacho, J. M. Soler, and P. Ordejon, Phys. Rev. B 61, 13639 (2000).

[70] J. Neugebauer and M. Scheffler, Phys. Rev. B 46, 16067 (1992).

[71] J. A. Stroscio, D. T. Pierce, A. Davies, and R. J. Celotta, and M. Weinert, Phys. Rev. Lett. 75, 2960 (1995). 